\title{
Prevalence of Exercise-Induced Bronchoconstriction Measured by Standardized Testing in Healthy College Athletes
}

\author{
David M Burnett PhD RRT AE-C, Steve Burns PhD, Samantha Merritt MSc, Jo Wick PhD, and \\ Matthew Sharpe MD
}

\begin{abstract}
BACKGROUND: Exercise-induced bronchoconstriction (EIB) can lead to long-term respiratory illness and even death. EIB prevalence rates are both high and variable in college athletes. Prevalence rates may be underestimated due to ineffective testing and screening. The purpose of this study was to investigate the prevalence of EIB in college athletes by a standardized EIB test that can be used on many college campuses. In addition, we assessed the usefulness of self-reporting EIB/asthma (1) history, (2) symptoms, and (3) respiratory medication obtained from a simple screening questionnaire for predicting an EIB-positive athlete. METHODS: A standardized EIB test and self-report questionnaire were administered to college athletes on 10 different sports teams. Information collected included pulmonary function (spirometry), expired gas analysis (maximal oxygen uptake), $\mathrm{CO}_{2}$ production, minute ventilation, $\mathrm{EIB} /$ asthma history, current symptoms, and medication use. RESULTS: Results showed that 34 of 80 athletes $(42.5 \%)$ were EIB-positive by standardized exercise testing. The majority (76.5 and $58.8 \%)$ of the 34 athletes who tested positive self-reported a negative history or no symptoms, respectively. Also, 79.4\% of the athletes who tested positive for EIB reported not using a respiratory medication. There were no significant differences in a positive EIB test when assessing interactions for history $(P=.93)$, current symptoms $(P=.12)$, or respiratory medication use $(P=.66)$. CONCLUSIONS: A high proportion of college athletes tested positive for EIB when using a standardized test. Positive history, current symptoms of EIB/asthma, and respiratory medication use were not predictive of a positive test. Many EIB-positive athletes are not using a respiratory medication. More work is needed to develop an effective screening tool and improve education for EIB in college athletes. Key words: bronchoconstriction; respiratory; asthma; exercise; sports; athletes. [Respir Care 2016;61(5):571-576. (C) 2016 Daedalus Enterprises]
\end{abstract}

\section{Introduction}

Exercise-induced bronchoconstriction (EIB) can cause intermittent inflammation and constriction of the airways,

\footnotetext{
Drs Burnett, Wick, and Sharpe are affiliated with the University of Kansas Medical Center, Kansas City, Kansas. Dr Burns and Ms Merritt are affiliated with the University of Central Missouri, Warrensburg, Missouri.

This work was supported by the University of Kansas Medical Center School of Health Professions. The authors have disclosed no conflicts of interest.

Correspondence: David M Burnett PhD RRT AE-C, Respiratory Care Education, Mail Stop 1013, University of Kansas Medical Center, 3901 Rainbow Boulevard, Kansas City, KS 66160. E-mail: dburnett@kumc.edu.
}

DOI: $10.4187 /$ respcare.04493 characterized by symptoms of chest tightness, wheezing, coughing, and unusual shortness of breath. Exercise and physical training can trigger bronchoconstriction, causing symptoms of EIB in $80-90 \%$ of people with asthma, ${ }^{1}$ and it has been shown that $10 \%$ of the population without asthma may experience symptoms related to EIB..$^{2,3}$ Moreover, previous studies reported that EIB was 3-5 times more prevalent in athletes when compared with the general population. ${ }^{4-6}$

Morbidity associated with EIB is speculated to originate from reoccurring episodes of inflammation during repeated bouts of exercise, leading to airway remodeling and chronic irreversible airway obstruction. ${ }^{7,8}$ More importantly, the potential for a severe episode of EIB leading to mortality has been noted in athletes. A 7-y study by Becker et $\mathrm{al}^{9}$ reported 61 EIB-related deaths during competition or practice, with $81 \%$ of these deaths occurring in athletes $<21$ y old. 


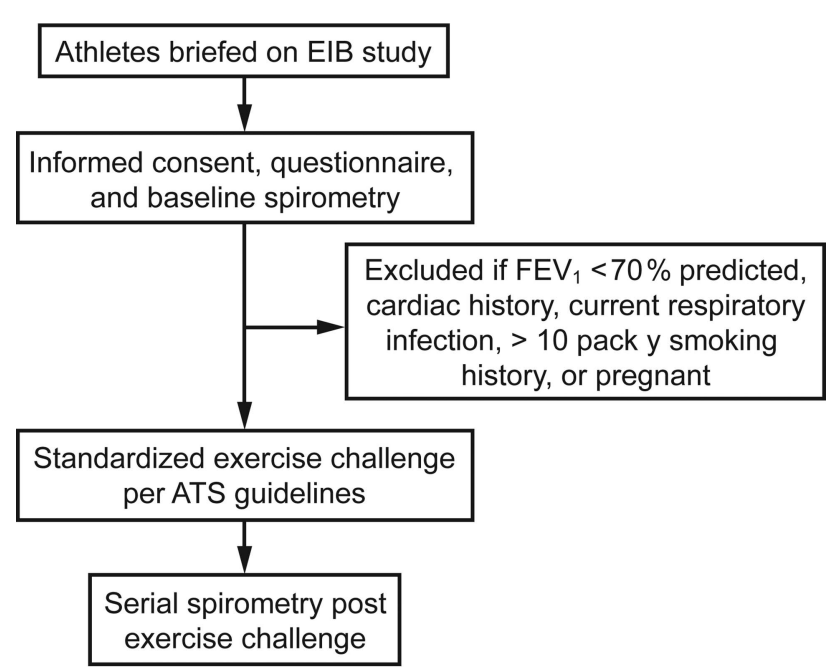

Fig. 1. Flow chart.

There is a need for more studies that adhere to a rigorous standardized EIB test protocol in order to ensure an accurate diagnosis of EIB for reporting on prevalence rates and associations with screening tools..$^{10,11}$ The primary aim of this study was to determine the prevalence of EIB in a cohort of Division II college athletes using a standardized EIB test protocol that could be duplicated on most college campuses. We also investigated any associations between a positive EIB test and a potential screening tool (questionnaire) that included a past history of EIB/asthma, current symptoms, or use of a respiratory medication. We hypothesized that we would find a robust proportion of college athletes with EIB when using a standardized EIB test, regardless of their past history, current symptoms, or use of respiratory medications.

See the Related Editorial on Page 713

\section{Methods}

\section{Participants and Study Design}

Participants in this study were selected from male and female athletes in 10 different sports teams at the University of Central Missouri during the 2014-2015 academic year. Exclusion criteria included a baseline $\mathrm{FEV}_{1}<70 \%$ of predicted, cardiac history, current respiratory infection, $>10$-pack-year history of smoking, or pregnancy. Athletes were recruited, between September 2014 and April 2015, at routine team meetings. Interested athletes received a brief explanation of the protocol and were given the opportunity to participate in the study (Fig. 1). All participation was voluntary, and a written informed consent was completed by each athlete before any data collection. The

\section{QUICK LOOK}

\section{Current knowledge}

Prevalence of exercise-induced bronchoconstriction (EIB) in college athletes can be both high and variable. College athletes do not accurately recognize or report EIB symptoms. College athlete self-reporting of EIB/asthma has not been shown to be predictive of a positive EIB test.

\section{What this paper contributes to our knowledge}

This study supports the high prevalence rate of EIB in college athletes when diagnosed by a standardized EIB test. Self-reporting of history, symptoms, and respiratory medication use does not predict a positive EIB test. A rigorous standardized EIB test can be performed on college campuses to help screen for EIB-positive athletes.

study was approved by both the Kansas University and University of Central Missouri institutional review boards.

\section{Study Protocol}

Participants who consented to participate in the study were scheduled for a single visit to complete a questionnaire and standardized EIB testing. The questionnaire consisted of 16 questions relating to respiratory and allergy history, symptoms, and medications. A history of asthma was defined by a previous diagnosis from a physician. All questions have been previously included in a health survey developed by the United States Olympic Committee. In addition, 6 questions relating to EIB symptoms and athletes' concern about EIB affecting sports performance were presented in the format of a 5-point Likert scale. An answer of $\geq 2$ (some of the time) on the Likert scale was considered as a positive response. The questionnaire was explained by a member of the research team and completed by all athletes before performing a standardized EIB test.

Each participant performed a standardized EIB test, including baseline spirometry, treadmill exercise challenge, and serial spirometry post-exercise challenge according to American Thoracic Society guidelines. ${ }^{12}$ Before spirometry testing, all asthma medications, including short-acting bronchodilators and long-acting bronchodilators, were withheld for 8 and $24 \mathrm{~h}$, respectively. At least 2 acceptable and repeatable $\mathrm{FEV}_{1}$ maneuvers were performed at baseline before a treadmill exercise challenge. An exercise bronchoprovocation challenge was performed on a treadmill after collecting the baseline spirometry. Before step- 
ping onto the treadmill, participants were fitted and acclimated to a mouthpiece, nose clip, and headgear (Hans Rudolph, Inc., Shawnee, Kansas) for gas analysis, including minute ventilation $\left(\dot{\mathrm{V}}_{\mathrm{E}}\right), \mathrm{CO}_{2}$ production, and $\mathrm{O}_{2}$ consumption. Participants began walking at a brisk pace on a treadmill while breathing dry air $\left(<10 \mathrm{mg} \mathrm{H}_{2} \mathrm{O} / \mathrm{L}\right)$. The protocol involved a rapid increase in intensity (grade and speed) over 2-4 min to achieve a $\dot{V}_{E}$ of at least 17.5-21 times the baseline $\mathrm{FEV}_{1}$ and $80-90 \%$ of the target heart rate $(\sim 220-$ age in years). Once this intensity was achieved, the participants maintained the high level of exercise for another 4-6 min. Serial spirometry was performed immediately after and 5, 10, 15, 20, 25, and $30 \mathrm{~min}$ after the exercise challenge. Two acceptable and reproducible spirometry measures were collected at each time interval, with the highest acceptable value recorded. The criterion for a positive test was a $\geq 10 \%$ fall in $\mathrm{FEV}_{1}$ from the baseline measurement.

\section{Statistical Analysis}

The primary aim was to determine the prevalence of EIB in a cohort of Division II college athletes using standardized EIB testing. Any associations between a positive EIB test and a past history of EIB/asthma, current symptoms, or use of asthma medication were also assessed. Descriptive statistics were used to describe group characteristics. Numbers and proportions were used to explain self-reported data (history of EIB or asthma, symptoms, and medication use) and standardized EIB testing results in college athletes. Pearson chi-square tests were used to analyze interactions between a positive EIB test and a past history of EIB/asthma, current symptoms, or use of asthma medication. All analyses were 2-tailed with $\alpha=0.05$ and performed with SPSS version 15.0 (SPSS, Chicago, Illinois).

\section{Results}

Eighty-five athletes from 10 different sports completed the questionnaire and standardized EIB test. Participant characteristics are shown in Table 1. Overall, participants were healthy and young and had normal lung function. Five athletes had a baseline $\mathrm{FEV}_{1}<70 \%$ of predicted and did not undergo an exercise challenge. All 5 athletes were referred to their physician for follow-up.

All athletes reached their mean target heart rate and $\dot{V}_{\mathrm{E}}$ (Table 2). Moreover, all athletes reached their target $\dot{\mathrm{V}}_{\mathrm{E}}$ and heart rate and had a normal ( $>80 \%$ of predicted $\mathrm{FEV}_{1}$ ) at baseline. Thirty-four of 80 athletes $(42.5 \%)$ tested positive when undergoing a standardized EIB test (Table 2). The EIB-positive group mean for percent-predicted FVC remained normal and without any clinically important change from baseline to post-exercise challenge, (96\% pre-
Table 1. Demographic Data

\begin{tabular}{lc}
\hline \hline \multicolumn{1}{c}{ Variables } & Values \\
\hline Participants, $N$ & 80 \\
Sex, \% male & 56 \\
Age, mean (range) & $20(18-23)$ \\
History of asthma or EIB, $n$ & 17 \\
Symptoms of asthma or EIB, $n$ & 22 \\
Use of a respiratory medication, $n$ & 17 \\
Baseline FEV & , mean \pm SD \% predicted \\
& \\
\hline EIB $=$ exercise-induced bronchoconstriction & \\
\hline
\end{tabular}

Table 2. EIB Testing Data

\begin{tabular}{lcc}
\hline \hline \multicolumn{1}{c}{ Variable } & $\begin{array}{c}\text { EIB-Positive } \\
\text { Athletes } \\
(n=34)\end{array}$ & $\begin{array}{c}\text { EIB-Negative } \\
\text { Athletes } \\
(n=46)\end{array}$ \\
\hline $\mathrm{FEV}_{1}$ at baseline, $\mathrm{L}$ & $3.83 \pm 0.81$ & $3.97 \pm 0.88$ \\
$\mathrm{FEV}_{1}$ at baseline, \% predicted & $93.3 \pm 13.9$ & $91.3 \pm 13.0$ \\
$\mathrm{Change}$ in $\mathrm{FEV}$ & & $\%$ change \\
$\quad$ from baseline & $-16.9 \% \pm 5.6$ & $4.2 \pm 3.7$ \\
Maximum heart rate, beats/min & $174 \pm 7.2$ & $174 \pm 6.5$ \\
Maximum $\dot{\mathrm{V}}_{\mathrm{E}}, \mathrm{L} / \mathrm{min}$ & $89 \pm 29.5$ & $92.5 \pm 29.3$ \\
& & \\
\hline $\begin{array}{l}\text { Values are mean } \pm \mathrm{SD} . \\
\dot{\mathrm{V}}_{\mathrm{E}}=\text { minute ventilation }\end{array}$ & & \\
\hline
\end{tabular}

exercise vs $92 \%$ post-exercise). However, the EIB-positive group mean $\mathrm{FEV}_{1}$ as a proportion of $\mathrm{FVC}$ $\left(\mathrm{FEV}_{1} / \mathrm{FVC}\right)$ indicated a clinically important decrease from $83 \%$ pre-exercise to $71 \%$ post-exercise. A large proportion, 26 of 34 (76.5\%), of those testing positive did not report a history of EIB or asthma (Fig. 2). More than half, 20 of $34(58.8 \%)$, of the 34 EIB-positive athletes did not report any symptoms of EIB or asthma (Fig. 2). A large proportion, 27 of 34 (79.4\%), of the EIB-positive athletes did not report using a respiratory medication (Fig. 2).

Interactions including past history, current symptoms, and respiratory medication use were analyzed for predicting an athlete with a positive standardized EIB test. A positive EIB test was not affected by the athletes' history, current symptoms, or respiratory medication use. Conversely, $44 \%$ of the athletes with a negative history tested positive for EIB, which was greater than the $35 \%$ of athletes who reported a positive history (Fig. 3). Similarly, $48 \%$ of athletes reporting to be asymptomatic tested positive for EIB compared to only $27 \%$ of athletes who reported symptoms (see Fig. 3). Also, a larger proportion of athletes $(44 \%)$ not using a respiratory medication tested positive compared with $41 \%$ of the athletes on an asthma medication (see Fig. 3). 


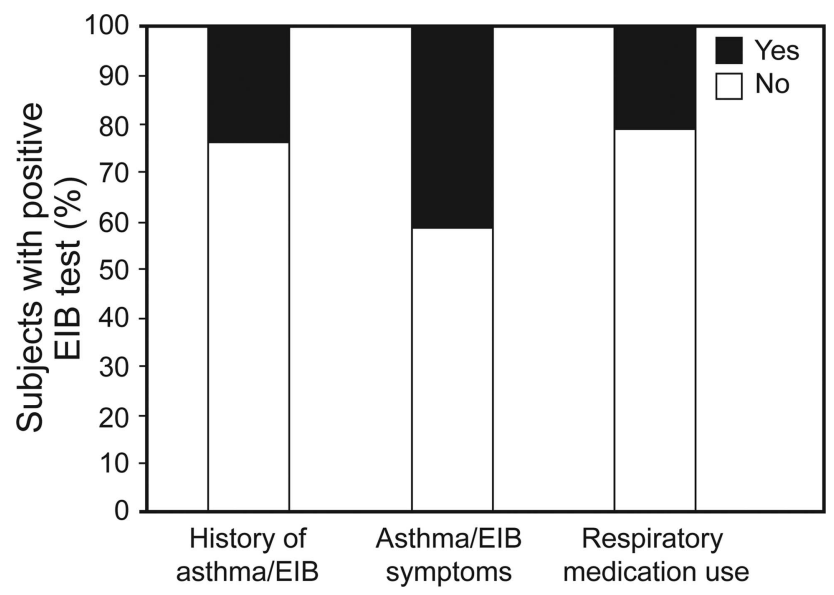

Fig. 2. Proportion of athletes who tested positive $(n=34)$ for exercise-induced bronchoconstriction (EIB) reporting a history of asthma/EIB, asthma/EIB symptoms, and respiratory medication use.

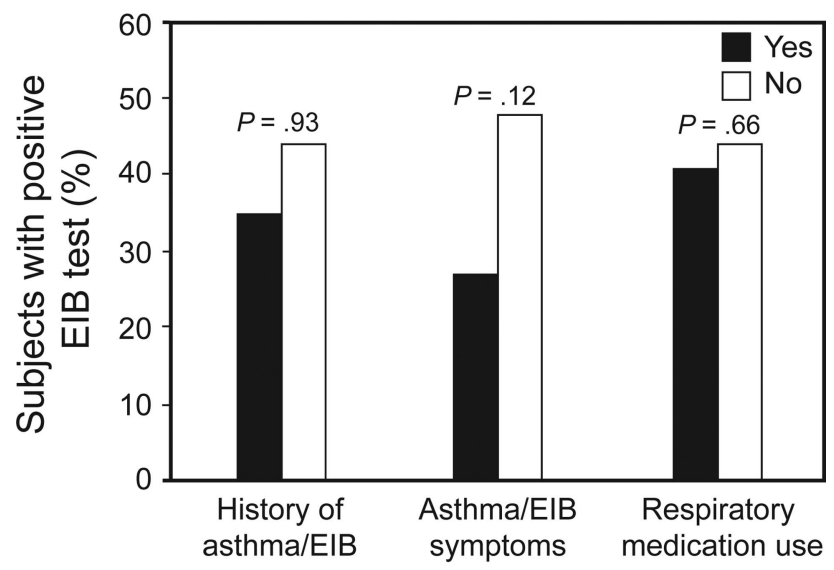

Fig. 3. Interactions between a positive exercise-induced bronchoconstriction (EIB) test with reported asthma/EIB history, asthma/EIB symptoms, and respiratory medication use.

\section{Discussion}

\section{Prevalence}

The results of this study are consistent with previous studies reporting a high prevalence of EIB in athletes when using the eucapnic voluntary hyperventilation testing modality.4,5,13,14 However, another study showed a low prevalence rate among college athletes when using the eucapnic voluntary hyperventilation testing method. ${ }^{15}$ Regardless of the variability seen with the prevalence of EIB in athletic populations, a previous workgroup reported a substantial EIB prevalence $(30-70 \%)$ in athletic populations using a variety of diagnostic methods. ${ }^{3}$ Given the significant prevalence of EIB reported in most studies it is critical to pursue an accessible testing method for accurately diagnosing athletes once they are screened for potential EIB. In the current study, an 80 of 85 (94\%) success rate was achieved while adhering to a strict standardized EIB testing protocol that may be duplicated at many college institutions.

\section{Prevalence and History}

The data in this study showed that there was not a significant difference in a positive EIB test between athletes with or without a previous history of EIB or asthma. However, the data indicated that a larger proportion of athletes with a negative history tested positive compared with those who reported a history of EIB or asthma. Our results are also consistent with previous studies reporting a lack of association between college athletes who were EIB-positive by objective testing with a previous history of EIB or asthma. ${ }^{4,5}$ Parsons et $\mathrm{al}^{5}$ showed that only $14 \%$ of college athletes who were EIB-positive had a previous history of EIB or asthma. Dickinson et $\mathrm{al}^{4}$ showed that only $27 \%$ of EIB-positive elite athletes reported a history of EIB or asthma. Our study also revealed that a small proportion $(23.5 \%)$ of college athletes who tested positive reported a history of EIB or asthma. Together, these findings suggest that detecting EIB in athletic populations can be difficult, and proper diagnostic screening has serious clinical relevance.

\section{Prevalence and Symptoms}

There was no significant difference in a positive EIB test between athletes with or without previous symptoms of EIB or asthma. As with history, the data were concerning, since a larger proportion of athletes reporting to be asymptomatic tested positive compared with those who reported symptoms of EIB or asthma. Our results are similar to those of previous studies suggesting that self-reported symptoms are not associated with testing positive when performing a standardized EIB test. ${ }^{5,15-17}$ This is clinically relevant from both an overdiagnosis and underdiagnosis perspective. A previous report indicated that the majority of physicians may overdiagnose and treat EIB with short-acting $\beta$ agonists based on symptoms alone, without assessing the athlete during an exercise challenge. ${ }^{18}$ Therefore, athletes may be using a short-acting $\beta$ agonist medication for months or even years without any results of measurable airway reactivity. On the other hand, EIB may be underdiagnosed for a number of reasons. Almost half (28 of 58) of the athletes who reported to be asymptomatic in our study were EIB-positive during the standardized EIB test. Many athletes may fail to report symptoms suggestive of EIB and not seek medical care to undergo a formal diagnosis. This may be attributed to the athlete's lack of awareness for symptoms and their association to 
EIB. Rather than recognizing the potential of having EIB, athletes may consider themselves to be in poor condition or believe they are having a bad training day. As a result, athletes potentially may continue to experience silent episodes of EIB. Together, these findings suggest that specialists in respiratory care may need to do a better job at teaching awareness of EIB to athletes, coaches, trainers, and parents. As previously mentioned, this can be clinically relevant, since a vast majority (81\%) of EIB deaths were in the college athlete age group or younger. ${ }^{9}$

\section{Prevalence and Respiratory Medication}

There was no significant difference in a positive EIB test between athletes using or not using a respiratory medication. Moreover, only $20 \%$ of the athletes who tested positive in this study reported using a respiratory medication. Evidence-based guidelines recommend the prophylactic use of a short-acting bronchodilator ( $\beta_{2}$ agonists) shortly before exercise as the main therapeutic treatment for EIB. ${ }^{19}$ Furthermore, the National Athletic Trainers Association guidelines suggest that athletic trainers in direct contact with athletes should understand the use, misuse, and abuse of $\beta_{2}$ agonists. ${ }^{20}$ Although guidelines support the use of $\beta_{2}$ agonists to minimize or prevent symptoms of asthma, patients with asthma may not adhere to their prescribed medication treatment. ${ }^{21}$ These findings may be related to a number of issues, including the lack of (1) awareness about EIB among sports teams and personnel, (2) education for athletes and parents, and (3) accurate screening and diagnosis of athletes. More work is needed to determine the barriers for the non-use of asthma medications in athletes.

Our study is limited due to its use of a convenience sample of athletes from a single university. A larger sample size could allow for the investigation of differences in prevalence among different sports. We did not test athletes from all sports, so our data may not be representative of athletes on all teams. Larger studies are needed to provide a better generalization of the college athletic population and investigate any differences between sports.

\section{Conclusions}

This study showed that a standardized EIB test can be performed on a college campus to accurately diagnose college athletes. A large proportion of athletes tested positive, and there were no significant differences found in EIB-positive athletes when assessing interactions for history, symptoms, or respiratory medication. This suggests that a prior history or self-reporting of symptoms is unreliable when attempting to accurately diagnose EIB in college athletes. More work is needed to evaluate the prevalence of EIB in college athletes while using an accessible and standardized test. In addition, future studies are needed to evaluate the clinical importance of an accurate and simple screening tool that can stratify at-risk athletes for standardized EIB testing. Accurately predicting student-athletes who test positive for EIB may help to moderate any unnecessary morbidity and mortality.

\section{ACKNOWLEDGMENTS}

We thank the subjects who participated in the study. Also, we thank the sports medicine team and coaches for allowing us to work with the athletes.

\section{REFERENCES}

1. McFadden ER Jr, Gilbert IA. Exercise-induced asthma. New Engl J Med 1994;330(19):1362-1367.

2. Gotshall RW. Exercise-induced bronchoconstriction. Drugs 2002; 62(12):1725-1739.

3. Weiler JM, Bonini S, Coifman R, Craig T, Delgado L, Capão-Filipe M, et al. American Academy of Allergy, Asthma and Immunology Work Group report: exercise-induced asthma. J Allergy Clin Immunol 2007;119(6):1349-1358.

4. Dickinson J, McConnell A, Whyte G. Diagnosis of exercise-induced bronchoconstriction: eucapnic voluntary hyperpnoea challenges identify previously undiagnosed elite athletes with exercise-induced bronchoconstriction. Br J Sports Med 2011;45(14):1126-1131.

5. Parsons JP, Kaeding C, Phillips G, Jarjoura D, Wadley G, Mastronarde JG. Prevalence of exercise-induced bronchospasm in a cohort of varsity college athletes. Med Sci Sports Exerc 2007;39(9):1487-1492.

6. Rundell KW, Jenkinson DM. Exercise-induced bronchospasm in the elite athlete. Sports Med 2002;32(9):583-600.

7. Bousquet J, Jeffery PK, Busse WW, Johnson M, Vignola AM. Asthma. From bronchoconstriction to airways inflammation and remodeling. Am J Respir Crit Care Med 2000;161(5):1720-1745.

8. Tiddens H, Silverman M, Bush A. The role of inflammation in airway disease: remodeling. Am J Respir Crit Care Med 2000;162(2 Pt 2):S7-S10.

9. Becker JM, Rogers J, Rossini G, Mirchandani H, D’Alonzo GE Jr. Asthma deaths during sports: report of a 7-year experience. J Allergy Clin Immunol 2004;113(2):264-267.

10. Stickland MK, Spooner CH, Dryden DM, Rowe BH. The need for standardization in exercise challenge testing for exercise-induced asthma/bronchoconstriction. J Allergy Clin Immunol 2010;126(4): 878-880.e6.

11. Weiler JM, Hallstrand TS, Parsons JP, Randolph C, Silvers WS, Storms WW, Bronstone A. Improving screening and diagnosis of exercise-induced bronchoconstriction: a call to action. J Allergy Clin Immunol Pract 2014;2(3):275-280.e7.

12. Parsons JP, Hallstrand TS, Mastronarde JG, Kaminsky DA, Rundell KW, Hull JH, et al. An official American Thoracic Society clinical practice guideline: exercise-induced bronchoconstriction. Am J Respir Crit Care Med 2013;187(9):1016-1027.

13. Holzer K, Anderson SD, Douglass J. Exercise in elite summer athletes: Challenges for diagnosis. J Allergy Clin Immunol 2002;110(3): 374-380.

14. Mannix ET, Roberts MA, Dukes HJ, Magnes CJ, Farber MO. Airways hyperresponsiveness in high school athletes. J Asthma 2004; 41(5):567-574.

15. Parsons JP, Cosmar D, Phillips G, Kaeding C, Best TM, Mastronarde JG. Screening for exercise-induced bronchoconstriction in college athletes. J Asthma 2012;49(2):153-157. 
16. Holzer K, Brukner P. Screening of athletes for exercise-induced bronchoconstriction. Clin J Sport Med 2004;14(3):134-138.

17. Rundell KW, Im J, Mayers LB, Wilber RL, Szmedra L, Schmitz HR. Self-reported symptoms and exercise-induced asthma in the elite athlete. Med Sci Sports Exerc 2001;33(2):208-213.

18. Parsons JP, O'Brien JM, Lucarelli MR, Mastronarde JG. Differences in the evaluation and management of exercise-induced bronchospasm between family physicians and pulmonologists. J Asthma 2006;43(5):379-384.

19. National Asthma Education and Prevention Program. Expert panel report 3 (EPR-3): guidelines for the diagnosis and management of asthma-summary report 2007. J Allergy Clin Immunol 2007;120(5 Suppl):S94-S138

20. Miller MG, Weiler JM, Baker R, Collins J, D'Alonzo G. National Athletic Trainers' Association position statement: management of asthma in athletes. J Athl Train 2005;40(3):224-245.

21. Spector SL, Kinsman R, Mawhinney H, Siegel SC, Rachelefsky GS, Katz RM, Rohr AS. Compliance of patients with asthma with an experimental aerosolized medication: implications for controlled clinical trials. J Allergy Clin Immunol 1986;77(1 Pt 1):65-70.

This article is approved for Continuing Respiratory Care Education credit. For information and to obtain your CRCE

(free to AARC members) visit

www.rcjournal.com 